\title{
La Falta de Reconocimiento de los Estándares Internacionales sobre Prisión Preventiva en la Justicia Uruguaya*
}

Este comentario, junto a los

documentos que analiza, está disponible en www.anuariocdh.uchile.cl

Diego Camaño Viera**

\section{Introducción}

$\angle$ a incorporación normativa de diversos instrumentos de derechos humanos ha sido una práctica histórica del Uruguay, que se ha posicionado entre los países con mayor cantidad de ratificaciones de tratados sobre la materia. Sin embargo, esta práctica no ha generado cambios sustanciales en el ejercicio del poder y en la aplicación del derecho puertas adentro, tanto a nivel ejecutivo como legislativo y judicial.

Por otra parte, esta brecha entre la incorporación formal y la efectividad real de los derechos humanos implica una frustración de la expectativa de universalidad del derecho internacional. Si el derecho internacional se ha inspirado en la idea del "ciudadano del mundo" mediante el reconocimiento de una serie de derechos a cualquier persona -independientemente del Estado que ejerza jurisdicción-, entonces resulta evidente que el Uruguay, al aplicar un status jurídico diferenciado ha reducido el umbral de protección del derecho internacional. En la práctica interna esto se traduce en la falta de aplicación de los estándares internacionales.

El caso de la prisión preventiva es paradigmático. La justicia uruguaya ha prestado oídos sordos a los textos que regulan los límites al encarcelamiento cautelar ${ }^{1}$, así como también a los estándares establecidos por los órganos encargados de la supervisión de los tratados. Tanto los informes de la Comisión Interamericana [en adelante $\mathrm{CIDH}$ ] como las sentencias de la Corte Interamericana de Derechos Humanos [en adelante $\mathrm{CtIDH}$ ] son un área inexplorada por parte de los tribunales locales, que siguen aplicando el derecho con la mira puesta exclusivamente en el estrecho campo de su producción doméstica.

Esto implica no sólo una grave afrenta al auténtico valor jurídico del derecho internacional (que de este modo queda reducido en el ámbito interno a un "no derecho") sino, ante todo, una fuente de graves violaciones a los derechos humanos de los detenidos, que por el sólo hecho de ser juzgados en el Uruguay se ven privados del reconocimiento de derechos fundamentales (por ejemplo, el derecho a no ser sometido a un encarcelamiento indeterminado); o deben sufrir la lisa y llana destrucción del principio de inocencia, toda vez que el legislador -sustituyendo ilegítimamente al juez- establece la prisión preventiva obligatoria para todos los delitos cuya pena mínima legal sea de al menos dos años de penitenciaría.

\footnotetext{
* El presente trabajo fue realizado sobre la base de un estudio más amplio, titulado "Límites normativos de la duración de la prisión preventiva", publicado en la Revista de Derecho Penal Nº 16. FCU, Montevideo, 2006.

** Abogado, Profesor Adjunto de Derecho Penal de la Facultad de Derecho de la Universidad de la República, miembro del Consejo Directivo del Instituto de Estudios Legales y Sociales del Uruguay (IELSUR).

1 Convención Americana sobre Derechos Humanos [en adelante CADH] y Pacto Internacional de Derechos Civiles y Políticos [en adelante PIDCP].
} 
Ciertamente, existen derechos reconocidos en instrumentos internacionales cuya justiciabilidad sólo ha tenido hasta el presente una aceptación teórica, como es el caso de los derechos económicos, sociales y culturales [en adelante DESC]. Y ello sin perjuicio de la reciente jurisprudencia interamericana en diversos casos sobre comunidades indígenas, en los que se han registrado avances importantes en este sentido ${ }^{2}$.

Se ha dicho con razón ${ }^{3}$ que no hay una diferencia estructural entre los DESC y los derechos civiles y políticos [en adelante DCP], dado que ambos implican prestaciones positivas y negativas a cargo del Estado. Sin embargo, pocas esperanzas pueden cifrarse en un pleno reconocimiento de los DESC si ni siquiera están dadas las condiciones para la protección de los DCP, que al día de hoy siguen constituyendo el "núcleo duro" de los derechos humanos.

En efecto, si los Estados no son capaces de respetar la vigencia de los DCP, poco cabe esperar del "futuro de los DESC". Por esta misma razón, bien vale la pena arrojarle a los primeros la tarea de abrir el camino a la justiciabilidad de los segundos, y de este modo comenzar a materializar la más importante aspiración del discurso sobre derechos humanos: su reconocimiento universal, indivisible e interdependiente.

Obsérvese que en el caso del encarcelamiento preventivo los instrumentos internacionales regulan nada menos que garantías judiciales, es decir, aquellas que debieran ser observadas por los jueces. En efecto, son ellos quienes tienen reservada la potestad exclusiva de decir la "última palabra" sobre su aplicación, determinando si "valen o no" en el caso concreto. En materia de prisión preventiva esto implica nada menos que decidir sobre la libertad de las personas.

Esta peculiaridad de las garantías judiciales, que no se presenta en otros derechos ${ }^{4}$, hace que la falta de reconocimiento de los estándares internacionales sea, en esta materia, particularmente preocupante.

Estas razones son por demás suficientes para justificar un estudio como el presente, comprensivo de la jurisprudencia sobre prisión preventiva de los Tribunales de Apelaciones en lo Penal [en adelante TAP] y de la Suprema Corte de Justicia [en adelante SCJ] entre 1989 y $2006^{5}$.

En él veremos cómo los estándares locales se contraponen en forma abierta, directa y sistemática con los textos internacionales y los estándares establecidos por los órganos encargados de supervisar su aplicación.

\section{La exigencia: decisiones consistentes y racionalmente aceptables}

En un Estado democrático, si los fallos judiciales quieren satisfacer la pretensión de legitimidad del derecho, deben presentarse como decisiones consistentes y ser racionalmente aceptables ${ }^{6}$.

2 Sólo como ejemplo, pueden citarse los casos Masacre Plan de Sánchez y Comunidad Mayagna tramitados ante la $\mathrm{CtIDH}$.

3 Abramovich, Víctor y Courtis, Christian: "Hacia la exigibilidad de los derechos económicos, sociales y culturales. Estándares internacionales y criterios de aplicación por los tribunales locales" en La aplicación de los tratados sobre derechos humanos por los tribunales locales, CELS-Ediciones Del Puerto, Bs. As., 2004, pp. 286 y 287.

4 Por ejemplo, los propios DESC, cuya realización depende en primer lugar del respeto por parte de otras autoridades públicas, principalmente ejecutivas.

5 En materia excarcelatoria las resoluciones de los jueces sólo son revisables por un TAP, competente por vía de apelación. Sin perjuicio de ello, la ley 17.726 de 26/12/2003 confirió competencia a la SCJ para "conceder la excarcelación provisional por gracia, atendiendo a la preventiva ya sufrida o a la excesiva prolongación del proceso".

6 Habermas, Jürgen: Facticidad y validez. Sobre el derecho y el Estado democrático de derecho en términos de teoría del discurso, Editorial Trotta, Madrid, 1998, p. 267. 
Por un lado, el principio de seguridad jurídica reclama que las decisiones judiciales sean consistentes, en el marco del orden jurídico vigente (producto tanto de decisiones legislativas y judiciales anteriores como de aquellas tradiciones que conforman el derecho consuetudinario). A su vez, la pretensión de legitimidad del orden jurídico exige que dichos fallos se fundamenten de un modo racional, de forma tal que puedan ser aceptados por los miembros de la comunidad jurídica como decisiones racionales ${ }^{7}$.

Pues bien, partiendo de la base que el derecho internacional de los derechos humanos integra el orden jurídico vigente, toda decisión judicial que se aparte de él constituye una decisión inconsistente, por falta de reconocimiento de una parte de ese orden jurídico.

Por otro lado, la aceptabilidad racional de la decisión judicial en la comunidad jurídica no puede darse sobre la base de un desconocimiento de los estándares internacionales establecidos por los órganos de supervisión de los tratados sobre derechos humanos.

En síntesis, la racionalidad de la administración de justicia consiste en que la aplicación del derecho tenga lugar "de forma internamente consistente y fundamentarse externamente de un modo racional, para asegurar simultáneamente la seguridad jurídica y la rectitud o corrección normativa" ${ }^{\prime}$.

\section{Los estándares de la jurisprudencia doméstica}

Analizaremos a continuación los principales estándares de la jurisprudencia doméstica que implican una falta de reconocimiento del derecho internacional.

\section{El carácter "programático" de la CADH}

Una clara muestra del desconocimiento del derecho internacional por la jurisprudencia doméstica es la consideración de la CADH como una "norma programática". En especial, el carácter programático de la garantía del "plazo razonable" vendría signado por su indefinición temporal, de lo que se sigue que la falta de una ley interna que establezca en abstracto un plazo máximo de duración de la prisión preventiva constituiría un escollo insalvable para la aplicación de dicha garantía 9 .

En esta línea, un fallo del TAP 3 se refiere al derecho internacional en términos particularmente hostiles: "No debe ni puede decretarse la defunción de la soberanía nacional en base a una normativa cuasi programática tanto por su indefinición sustantiva cuanto por la total imprecisión cronológica, para ejercitar una garantía jurisdiccional bien que no constitucional. Por otra parte, este Cuerpo que es un celoso defensor del sistema garantista tampoco ignora que la Convención Americana de Derechos Humanos $(\mathrm{CADH})$ plasmó en un tiempo sociopolítico pautado por la ausencia de garantías, lo cual indica que tuvo una precisa ratio legis o finalidad teleológica, sin desconocer otras realidades obvias a las cuales también comprende" ${ }^{\prime 10}$.

En primer término, resulta incomprensible la atribución de una concreta ratio legis a la CADH, y sorprende que éste sea un argumento para postular su desaplicación. Ello sumado al absurdo de

\footnotetext{
Habermas, op. cit., pp. 267 y 268.

8 Habermas, op. cit., p. 268.

9 TAP 1, Sent. 74/94, en Revista de Derecho Penal [en adelante RDP] No 11, p. 305; TAP 3, Sents. 222/94 y 212/96, en $R D P N^{\circ} 11$, pp. 306 y 309.

10 TAP 3, Sent. 230/94, en RDP No 11, pp. 306 y 307.
} 
sostener que la voluntad de los Estados fue la de crear dicho instrumento para aplicarlo exclusivamente en un particular momento histórico.

En segundo lugar, obsérvese que según esta jurisprudencia la aplicación de la CADH sería nada menos que la "partida de defunción" de la soberanía nacional, sin que resulte relevante considerar los derechos en juego (en especial, el derecho a la libertad). La ilegitimidad del argumento de la soberanía es evidente, pues de ser válido tornaría inaplicable al derecho internacional en su conjunto. En definitiva, según este criterio, la soberanía se erige en una "razón de Estado" antes que en una verdadera razón jurídica y la consecuencia directa de su invocación es el desconocimiento del derecho internacional como integrante del orden jurídico. Se trata de una decisión inconsistente, en función de dicha falta de reconocimiento.

\section{El criterio casuístico como impedimento}

Un segundo argumento de la jurisprudencia local para desaplicar la garantía del plazo razonable también se relaciona con la falta de una ley interna que determine en abstracto su período de duración. Pero en lugar de vincularse al carácter "programático" de la CADH, esa "falta de reglamentación interna", estableciendo un plazo fijo de duración de la prisión preventiva, sería un obstáculo por la imposibilidad de establecer dicho plazo en base al criterio "caso a caso", tal como lo sostiene la jurisprudencia internacional. En definitiva, desde este punto de vista, la falta de una ley interna que establezca un plazo fijo, sumada a la imposibilidad de aplicar un criterio casuístico, tornaría inaplicable la garantía.

Ya en 1968, la Corte Europea de Derechos Humanos [en adelante CtEDH] en el caso Wemhoff sostuvo la imposibilidad de traducir el concepto de plazo razonable en un número fijo de días, semanas, meses o años, estableciendo en su lugar la necesidad de ponderar la duración del encarcelamiento con un criterio casuístico, es decir, "caso a caso".

Paradójicamente, la jurisprudencia local ha invocado esa misma jurisprudencia de la CtEDH, pero no como justificación para ponderar si en el caso concreto el plazo de detención fue razonable (o no) sino, por el contrario, para postular la desaplicación de la garantía. En este sentido, se sostuvo que al no ser posible fijar un plazo en abstracto y no haberse dictado una ley que lo establezca con carácter general, la garantía no podría ser aplicada ${ }^{11}$.

De este modo, se ignora completamente que en el caso Giménez la CIDH ha establecido claramente que "Ios Estados miembros de la Convención no tienen la obligación de fijar un plazo fijo para la privación de libertad previa a la sentencia que sea independiente de las circunstancias de cada caso $^{\prime 12}$.

Por lo tanto, contrariamente a lo decidido por la jurisprudencia doméstica, la ausencia de una ley interna que establezca un período en abstracto no es un impedimento para hacer valer la garantía, pues el "plazo razonable" del encarcelamiento preventivo debe valorarse con un criterio casuístico.

Y ello afecta irremediablemente la aceptabilidad racional de la decisión, pues el argumento de la imposibilidad de establecer un plazo en abstracto no puede ser usado para descartar la aplicación de la garantía sino únicamente para justificar la necesidad de acudir a una valoración de la razonabilidad del tiempo de encarcelamiento transcurrido en cada caso.

11 TAP 1, Sent. 74/94, en RDP No 11, p. 305.

$12 \mathrm{CIDH}$, Caso Giménez, párr. 69. 


\section{La asimilación sustancialista entre prisión preventiva y pena}

Muy común en la jurisprudencia local es la introducción de valoraciones sustancialistas a la prisión preventiva, esto es, propias de las que corresponderían a la imposición de una pena.

De este modo, se desvirtúa la naturaleza cautelar del encarcelamiento preventivo, claramente afirmada en el ámbito internacional en casos como Giménez de la CIDH y Suárez Rosero de la $\mathrm{CtIDH}$.

En este sentido, el TAP 2 ha interpretado correctamente "la necesidad de evaluar el plazo que ha insumido el proceso, de manera casuística", tal como se desprende de la jurisprudencia de la $\mathrm{CtEDH}^{13}$. Sin embargo, acto seguido afirmó: "Es evidente que esa duración está en relación con la gravedad y complejidad de los delitos cometidos, del número de involucrados, de la necesaria investigación requerida para desentrañar la trama, de la cooperación de los justiciables"14. De este modo, se desvirtúa la naturaleza procesal de la prisión preventiva, haciendo jugar aspectos que tienen que ver con el fondo del asunto, tales como la gravedad del delito o la severidad de la pena legalmente establecida.

Por su parte, el TAP 1 sostuvo que "(el) plazo razonable de la norma internacional invocada debe estar referido a las conductas en examen y las normas punitivas aplicables"15.

En esta misma línea sustancialista, no es extraño encontrar pronunciamientos sobre las circunstancias del hecho e incluso la peligrosidad del detenido en resoluciones judiciales para denegar excarcelaciones ${ }^{16}$. En un caso se justificó la prisión preventiva en base a la "gravedad y complejidad de los delitos cometidos", sosteniendo que resulta inaceptable "exigir prisas y urgencias a la hora del esclarecimiento de eventos que se han cometido al amparo de una ingeniosa, como cuidada y razonada operativa" 17 .

Esta asimilación sustancialista entre prisión preventiva y pena ha dado lugar, en la práctica, a una grave disfunción: la aplicación de la prisión preventiva como pena anticipada. Y esto es justamente lo que el derecho internacional quiere prevenir al conferirle un carácter excepcional al encarcelamiento cautelar, evitando su asimilación con la pena.

Al introducir criterios de derecho penal sustancial (gravedad del delito y normas punitivas aplicables) para valorar un instituto procesal, la excarcelación opera de acuerdo a parámetros sustantivos y no procesales, como lo exige la normativa y jurisprudencia internacionales. Evidentemente, puede observarse aquí una gruesa violación al principio de inocencia, pues esas ponderaciones sustantivas implican un auténtico prejuzgamiento sobre aspectos propios de la sentencia de condena. Dichas consideraciones no pueden formar parte de la justificación de la decisión de excarcelación de una persona que todavía está siendo sometida a proceso, pues en tal calidad es titular del derecho a ser tratada como inocente.

Resulta por demás claro que todos los fallos que introducen este tipo de consideraciones sustantivas para valorar el "plazo razonable" de la prisión preventiva constituyen decisiones que no pueden ser aceptadas por la comunidad jurídica, por desconocer abiertamente el principio de inocencia, lo que afecta tanto su racionalidad como su consistencia.

\footnotetext{
13 Ver Cap. III.2. de este trabajo.

14 TAP 2, Sent. 345/03, en RDP N 15 , p. 457.

15 TAP 1, Sent. 218/02, en RDP $N^{\circ} 15$, p. 457.

16 TAP 2, Sent. 316/94, en RDP N $N^{\circ} 11$, p. 308.

17 TAP 2, Sent. 345/03, en RDP N $N^{\circ}$ 15, p. 457.
} 


\section{La prisión preventiva como regla}

Contrariamente al carácter excepcional del encarcelamiento cautelar, explícitamente establecido en el art. 9.3 del PIDCP e implícitamente previsto en el art. 7.5 de la CADH (según lo afirmado por la CIDH en Giménez y la CtIDH en Suárez Rosero), la jurisprudencia local ha establecido en diversos fallos que la prisión preventiva es la regla, siendo excepcional la procedencia de la excarcelación.

Para ello se trae a colación el art. 27 de la Constitución ${ }^{18}$, interpretado como una prohibición de excarcelación en delitos con pena mínima de penitenciaría ${ }^{19}$. Dicha prohibición se extraería a contrario sensu, ya que el texto establece simplemente una facultad de excarcelar en delitos con pena mínima de prisión y no una prohibición para los delitos con pena mínima de penitenciaría.

Sin embargo, se soslaya totalmente que las prohibiciones, en tanto limitaciones a la libertad personal, no pueden ser inferidas sino que sólo pueden surgir de un texto expreso que no existe en la especie, por lo que no hay contradicción alguna entre la letra constitucional y el derecho internacional. Y sabido es que en caso de duda sobre la preeminencia de la normativa internacional en relación con la constitucional, debe tenerse presente la regla de la primacía de la norma más favorable ${ }^{20}$, pues el derecho internacional de los derechos humanos no es una restricción sino, por el contrario, una ampliación de la protección constitucional.

Tampoco la jurisprudencia tiene en cuenta que el art. 72 de la Constitución contiene una "cláusula de derechos implícitos" -similar a la establecida en el propio art. 29.c de la CADH-, según la cual se reconocen como integrantes del texto constitucional todos aquellos derechos que no estén expresamente previstos pero que sean "inherentes a la personalidad humana o se [deriven] de la forma republicana de gobierno". Por medio de esta cláusula es posible atribuir a los derechos humanos rango constitucional ${ }^{21}$.

Por otra parte, también se deja totalmente de lado la aplicación del art. 27.1 de la Convención de Viena sobre el Derecho de los Tratados, según el cual: "Un Estado no podrá invocar las disposiciones de su derecho interno como justificación del incumplimiento de un Tratado". Si esto es válido para el derecho internacional general, mucho más lo es para el derecho internacional de los derechos humanos. Esto se debe a que, como ha dicho la $\mathrm{CtIDH}$ : "Ios tratados modernos sobre derechos humanos (...) no son tratados multilaterales de tipo tradicional, concluidos en función de un intercambio recíproco de derechos, para el beneficio mutuo de los Estados contratantes. Su objeto y fin son la protección de los derechos fundamentales de los seres humanos (...) Al aprobar estos tratados sobre derechos humanos, los Estados se someten a un orden legal dentro del cual ellos, por el bien común, asumen varias obligaciones, no en relación con otros Estados, sino hacia los individuos bajo su jurisdicción" 22 .

Lo que resulta sorprendente en la jurisprudencia local es que no advierte problemas interpretativos ni tampoco colisión alguna con el derecho internacional. Simplemente afirma un supuesto "obstáculo constitucional" para la excarcelación, omitiendo una ponderación del derecho a la

\footnotetext{
18 "En cualquier estado de una causa criminal de que no haya de resultar pena de penitenciaría, los Jueces podrán poner al acusado en libertad, dando fianza según la ley".

19 En el Uruguay la ley distingue la pena de penitenciaría (de 2 a 30 años) de la pena de prisión (de 3 a 24 meses).

$20 \mathrm{CADH}$, art. 29 lit. a y b; PIDCP, art. 52.

21 IELSUR (Instituto de Estudios Legales y Sociales del Uruguay): Estudio sobre armonización legislativa conforme los tratados de derechos humanos ratificados por Uruguay u otras normas legales con fuerza vinculante, pp. 81, 86, 92, 96. OACDH -IELSUR-PNUD, Uruguay, 2006.

22 CtIDH, Opinión Consultiva, OC 2/82, párr. 29 (destacado nuestro).
} 
libertad personal con relación al presunto interés de la persecución penal, así como cualquier referencia a los estándares internacionales.

Un pronunciamiento representativo del estado actual de la justicia uruguaya en este punto proviene del TAP 2: "La previsión constitucional del Pacto de San José de Costa Rica, por el cual todo encausado tiene derecho a la libertad provisional, cuando la tramitación del proceso excede del tiempo razonable, no configura en la sistemática procesal uruguaya, el principio o regla; por el contrario, cuando la pena a recaer es de penitenciaría, el designio constitucional obsta al otorgamiento de la libertad provisional (art. 27 de la Constitución) y sólo en casos excepcionales -que no es el presente- puede otorgarse la excarcelación" ${ }^{23}$.

El mismo criterio sostiene el TAP 1, pues si bien reconoce que "el art. 15 de la ley 15.737 puso en vigencia como Derecho Interno a la Convención de Derechos Humanos", acto seguido afirma que "en la interpretación de normas, lo cierto es que la jurisprudencia práctica ha recogido en numerosos pronunciamientos el criterio de la excepcionalidad para conceder libertades, que de otro modo se verían bloqueadas por la disposición constitucional"24.

Por lo tanto, mientras para el derecho internacional la regla general es la libertad y el encarcelamiento la excepción, en nuestra praxis judicial es exactamente al revés: la excarcelación constituye una excepción a la prisión preventiva.

De este modo se destruye el principio de inocencia y se permite que la prisión preventiva funcione como una pena anticipada de facto, que, como se dijo, es justamente lo que el derecho internacional pretende evitar.

\section{Los fallos de la SCJ y sus repercusiones en el Sistema Interamericano de Derechos Humanos}

Ya hemos señalado que, sin afectar la competencia para conceder excarcelaciones conferida a los Jueces y Tribunales de Alzada, la ley 17.726 de 26/12/2003 confirió a la SCJ la competencia de excarcelar en base a su poder de gracia, atendiendo a la prisión preventiva sufrida o a la excesiva duración del proceso ${ }^{25}$.

Resulta importante destacar que en un caso actualmente sometido a la CIDH, la SCJ denegó la libertad sin aplicar los estándares internacionales en materia de prisión preventiva. Así, las resoluciones $N^{\circ}$ 661, del 30 de marzo de 2005 y No 3374, del 24 de noviembre de 2006, denegaron la libertad provisional solicitada, pese a haberse invocado la violación del plazo razonable para la detención, y pese a la denuncia formulada ante la CIDH. Es más, la última sentencia fue posterior a la declaración de admisibilidad de la $\mathrm{CIDH}^{26}$ y no expresó fundamento alguno.

Por lo tanto, la justicia uruguaya aún no se ha percatado de que la aplicación del derecho internacional de los derechos humanos hace a la plena vigencia del Estado de Derecho. Además, tampoco parece haber advertido que este comportamiento compromete seriamente la responsabilidad internacional del Estado.

23 TAP 2, Sent. 316/94, en $R D P N^{\circ} 11$, p. 308, c. 431. En el mismo sentido, TAP 1, Sent. 147/95, en RDP $N^{\circ} 11$, p. 305, c. 426; TAP 3, Sent. 98/94, en $R D P N^{\circ} 11$, p. 304, c. 423.

24 TAP 1, Sent. 228/94, en RDP N 11 , p. 307, c. 430.

25 Ver nota al pie de página $\mathrm{N}^{\circ} 5$ de este trabajo.

${ }^{26} \mathrm{CIDH}$, Informe de admisibilidad No 35/06 de 14/3/06. Este es el primer caso uruguayo presentado ante el sistema interamericano por violación al plazo razonable de duración de la prisión preventiva. Al no haberse arribado a una solución amistosa, el próximo paso será el dictado del Informe sobre el fondo del asunto. 


\section{A modo de conclusión}

Con lo visto hasta ahora surge claro que la jurisprudencia local no ha dado aplicación a los estándares internacionales en materia de prisión preventiva. Por el contrario, aplica en el fuero interno un status jurídico devaluado con relación a la protección internacional.

En los casos en que se otorgaron libertades por exceso en la duración de la prisión preventiva, esto ha sido determinado por razones diferentes a las derivadas de la aplicación del derecho internacional de los derechos humanos. Se trata de situaciones que son abordadas como patológicas o excepcionales, donde las motivaciones de los fallos traslucen más bien razones sustantivas o de justicia material, generalmente para evitar un peor trato del imputado en relación con el condenado.

Sin embargo, la tendencia de fallos recientes incluso ha limitado esta posición, al afirmar por ejemplo que el cumplimiento de la mitad de la eventual pena no es argumento suficiente, o que razones procesales impiden un pronunciamiento previo a la sentencia de condena.

En definitiva, la invocación del "plazo razonable" no ha sido acompañada de la aplicación de los estándares internacionales en la materia sino que obedece a la necesidad de paliar situaciones aberrantes ocasionadas en el funcionamiento práctico de la justicia penal. La ausencia de toda mención al principio de inocencia y la inexistencia de desarrollos en torno al derecho a la libertad personal demuestran que la jurisprudencia no ha advertido la trascendencia de los derechos que se vulneran con la prisión preventiva. Además, la falta de invocación de las normas y estándares internacionales revela su nula influencia en la jurisprudencia doméstica. Por lo tanto, en los hechos, los límites al poder punitivo durante el enjuiciamiento están muy lejos de ser una realidad tangible de la justicia uruguaya, pese a estar establecidos normativamente. Ello redunda en una violación sistemática de los derechos de todos los imputados encarcelados y constituye un riesgo latente para aquellos que en un futuro lo serán.

En la práctica, la utilización indiscriminada de la prisión preventiva ha contribuido decididamente a la explosiva situación carcelaria, producto de un incremento desmesurado del número de personas privadas de libertad (que se ha duplicado en los últimos quince años) y del índice de presos sin condena (que es superior al $60 \%$ de la población carcelaria) ${ }^{27}$.

Para revertir esta situación a largo plazo es necesaria la adopción de medidas legislativas en materia de prisión preventiva; pero en lo inmediato es tarea primera de la justicia la protección efectiva de los derechos humanos, a través de la aplicación de las normas y estándares internacionales. Ello servirá no sólo para evitar la responsabilidad del Estado bajo el derecho internacional sino fundamentalmente para asegurar el respeto de los derechos de todas y cada una de las personas detenidas.

\footnotetext{
27 Según datos del ILANUD, en 1992 Uruguay tenía 3.037 presos, cifra que trepó a 4.012 en 1999. Actualmente cuenta con más de 7.000 presos. Ello arroja una tasa de encarcelamiento de más de 200 presos cada 100.000 hab. (una de las más altas de América Latina) y un crecimiento de más de un 100\% de la población carcelaria entre 1992 y 2007.
} 